\title{
Improved implementation of the transfer-to-the-continuum method for single-neutron knockout reactions and the validity of standard approximations
}

\author{
A. García-Camacho, ${ }^{*}$ R. C. Johnson, and J. A. Tostevin \\ Department of Physics, School of Electronics and Physical Sciences, University of Surrey, Guildford, Surrey GU2 7XH, United Kingdom
}

(Received 26 November 2004; published 26 April 2005)

\begin{abstract}
Approximations made in implementing the transfer-to-the-continuum model for single-nucleon knockout reactions on light target nuclei are investigated using more complete numerical calculations. The reliability of different proposed approximation schemes and their predicted cross sections are discussed. The results of the model calculations that use realistic descriptions of the distorting interactions entering the theory are also compared with available experimental data for reactions induced by ${ }^{15} \mathrm{C}$ and ${ }^{34} \mathrm{Si}$ secondary beams. These transitions, with different neutron orbital angular momenta, also have significantly different values of the neutron separation energy. The different approximation schemes are shown to agree most closely for the weakly bound and spatially extended, $l=0,{ }^{15} \mathrm{C}$ ground state transition. For an $l=2$ transition in ${ }^{34} \mathrm{Si}$, the approximation schemes are shown to be dependent on the nucleon separation energy. In all cases comparisons of even the most accurate implementation of the theory with the experimental data reveals deviations in both the magnitudes of the predicted integrated cross sections and their momentum distributions. Furthermore, the use of the different approximation schemes also produce quite significant effects on the shapes of the predicted momentum distributions and the integrated partial cross sections.
\end{abstract}

DOI: 10.1103/PhysRevC.71.044606

PACS number(s): 21.10.Jx, 24.10.-i, 25.60.Gc, 27.30.+t

\section{INTRODUCTION}

Single-nucleon knockout reactions induced by rare intermediate energy secondary beams are currently the object of considerable interest from both a theoretical and experimental point of view [1-4]. They permit the identification of nucleon level orderings and their associated spectroscopic strengths which can be compared with the predictions of modern structure theories. The utility of such reactions for the single-particle spectroscopy of exotic nuclei have been discussed extensively elsewhere, e.g. [5-7].

Several reaction models have now been developed with the aim of making detailed quantitative comparisons with the results of experiments. In this work we consider what has become known as the transfer-to-the-continuum (TC) model, as developed by Bonaccorso and Brink [8]. The model has its basis in the distorted wave Born approximation (DWBA) treatment of nucleon transfer reactions between heavy nuclear cores [9-12], supplemented by a semiclassical treatment of the entrance and exit channel distorted waves [13]. Because of the way that the coordinates of the heavy projectile core are treated the theory is not strictly DWBA, but is an approximation to DWBA.

Several TC model variants, each involving further simplifications in the evaluation of the TC approximation to the DWBA transfer amplitude, have been published. These have been motivated, in large part, by a wish to maintain nearanalytical forms for the reaction observables-specifically the nucleon removal cross section and the momentum distribution of the heavy projectile remnant. In the present paper we will

*Electronic address: camacho@df.unipi.it; Present address: Instituto Nazionale di Fisica Nucleare, Sezione di Pisa, I-56100 Pisa, Italy. look critically at these different approximation schemes by performing calculations of the TC amplitude in its more exact form. This necessitates a greater numerical effort. In this way we will check the accuracy of these often-used approximations. We will restrict discussion here to entrance and exit channel interactions with the target that are spin independent. An analysis of the effects of spin-dependent interactions with the target on the TC model predictions of cross sections, residue magnetic substate populations and excited residue decay-photon angular distributions will be reported elsewhere.

The main theoretical ingredients of the TC model are presented in Sec. II and the different approximation schemes are developed in Sec. III. In Sec. IV the choice of physical parameters and model interactions that enter the calculations are discussed. Sec. V then presents applications to specific single-neutron knockout reactions and clarifies the outcomes and the accuracy of the different approximation schemes of Sec. III, all calculated using a common set of physical model parameters.

\section{THEORETICAL BASIS OF THE MODEL}

The starting point for the transfer-to-the-continuum model of the breakup reaction is the one-step DWBA transition amplitude for the transfer of a light particle between discrete bound states in heavy nuclei [8]. The projectile nucleus is treated as the bound state of a heavy core nucleus and the transferred particle, which together with the heavy target constitute an effective three-body problem. The TC model [8] then considers the projectile breakup mechanism as a generalized particle transfer in which the final states are now the unbound continuum states of the transferred particle-target spectrum. The key steps of this development are summarized 
below. Throughout the present paper, the transferred particle is assumed to be a neutron.

\section{A. Semiclassical approach to particle transfer}

Within the one-step DWBA approach, the neutron transfer reaction differential cross section can be written $[10,13]$

$$
\frac{d \sigma}{d \Omega}=\frac{1}{\left(2 l_{1}+1\right)} \frac{\pi}{K^{2}} \frac{\mu_{i}}{\mu_{f}} \sum_{m_{1} m_{2}}\left|T_{l_{1} m_{1} l_{2} m_{2}}\right|^{2} .
$$

Here $l_{1}, m_{1}$ and $l_{2}, m_{2}$ are the orbital angular momentum quantum numbers of the transferred neutron in the initial and final bound states, $\mu_{i}$ and $\mu_{f}$ are the neutron-core and neutron-target reduced masses, and $K$ is the wave number of the projectile-target relative motion. Spin degrees of freedom have been suppressed. The transfer amplitude $T_{l_{1} m_{1} l_{2} m_{2}}$, upon applying the WKB approximation and the semiclassical orbit concept to the entrance and exit channel distorted waves [13], can be written, with $\hat{L}=\sqrt{2 L+1}$, as

$$
\begin{aligned}
T_{l_{1} m_{1} l_{2} m_{2}}(\widehat{\Omega})= & i^{m_{1}-m_{2}} \sum_{L} \hat{L} \exp \left(2 i \delta_{L}\right) \\
& \times A\left(L, l_{1} m_{1} l_{2} m_{2}\right) Y_{L m_{1}-m_{2}}(\widehat{\Omega}) .
\end{aligned}
$$

Here $\widehat{\Omega}$ denotes the scattering angles of the deflected core, $L$ is the relative angular momentum between the core and the target and $\delta_{L}$ the associated phase shift, the sum of nuclear and Coulomb scattering phase shifts. Finally, $A$ is a semiclassical transfer amplitude in which the core degrees of freedom enter only through the quantum number $L$, but not through any angular or other dependence. Calculation of the transfer process thus involves only the transferred neutron degrees of freedom. The explicit expression for $A$ is [10]

$$
A\left(L, l_{1} m_{1} l_{2} m_{2}\right)=\frac{1}{i \hbar} \int_{-\infty}^{\infty} d t\left\langle\Psi_{f}\left|V_{\mathrm{cn}}\right| \Psi_{i}\right\rangle,
$$

where $\Psi_{i}$ is the wave function of the neutron in the initial state, i.e., the bound-state solution of the Schrödinger equation for the neutron and core in potential $V_{\mathrm{cn}}$. Similarly, $\Psi_{f}$ is the neutron-target relative motion wave function in the final state with potential $V_{t n}$.

An explicit expression for this semiclassical transfer amplitude can be obtained upon making assumptions for the motion of the core. Near the distance of closest approach, the problem is simplified in the following ways. (i) The masses of the core and the target nuclei are assumed to be much larger than that of the transferred particle. (ii) The core is assumed to follow a straight-line trajectory $\vec{R}(t)$, with impact parameter $b=(L+1 / 2) / K$, and with a constant velocity. In this way the transfer between the core and target nuclei is transformed into a problem in which the neutron feels the effect of two potential fields in motion with respect to one another [14]. We describe this process using a target-centered reference frame with $z$ axis chosen in the incident beam direction and the $x$ axis in the plane of the core-target relative motion. The neutron position coordinate in this frame is $\vec{r}$ and hence $V_{\mathrm{cn}}$ in Eq. (3) has argument $\vec{r}-\vec{R}(t)$, Fig. 1 . The neutron transfer will take place in a localized region of the core straight-line trajectory, near the core-target distance of closest approach. In practice,

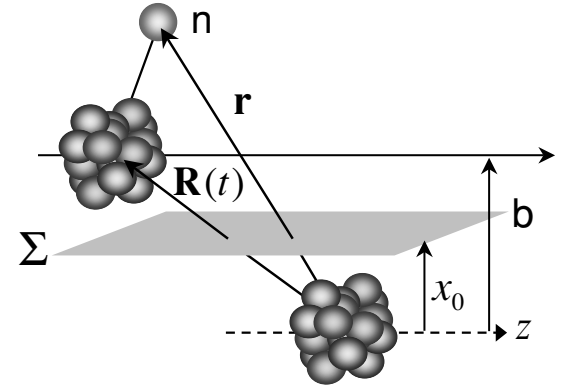

FIG. 1. Schematic of the core, target, and neutron three-body problem, showing the coordinate system used, the straight line core trajectory and the intervening $\Sigma$ surface in the external region of the neutron wave functions.

for the beam energies discussed here, the distance of closest approach is identified with the core-target impact parameter $b$, the Coulomb interaction being assumed not to modify the core trajectory [9].

Since our choice of reference frame is fixed at the target, a Galilean transformation is required to boost the initial state wave function. This is given by [15]

$$
\Psi_{i}(\vec{r}, t)=\phi_{l_{1} m_{1}}[\vec{r}-\vec{R}(t)] \exp \left[i\left(m \vec{v} \cdot \vec{r}-\epsilon_{i} t-m v^{2} t / 2\right) / \hbar\right],
$$

which includes the time dependence of the core coordinate. Here $\phi_{l_{1} m_{1}}$ is the bound state wave function of the neutron-core system in its rest frame. The final state does not require a Galilean boost, and hence

$$
\Psi_{f}(\vec{r}, t)=\phi_{l_{2} m_{2}}(\vec{r}) \exp \left[-i \epsilon_{f} t / \hbar\right] .
$$

It is now further assumed that the reaction is sufficiently peripheral that one can insert a planar surface $\Sigma$ between the core and target nuclei, that lies entirely in a region where the neutron potentials with the core and target, $V_{\mathrm{cn}}$ and $V_{\mathrm{tn}}$, are negligible, Fig. 1. In this case the transfer amplitude can be written $[9,10]$ with reference only to wave function values on $\Sigma$ as

$A\left(b, l_{1} m_{1} l_{2} m_{2}\right)=\frac{\hbar}{2 m i} \int_{-\infty}^{\infty} d t \int_{\Sigma} d \vec{S} \cdot\left(\Psi_{f}^{*} \nabla_{\vec{r}} \Psi_{i}-\Psi_{i} \nabla_{\vec{r}} \Psi_{f}^{*}\right)$.

Since the $\Sigma$ surface defines a region external to the neutron interactions, only the external, preasymptotic, analytic expressions for the initial and final state neutron wave functions are then required.

As stated earlier, this model was originally created for transfer reactions and then extended to breakup. At this point it can be seen that such a distinction enters through the form assumed for $\Psi_{f}$. In the transfer case a bound state wave function is used, whereas in breakup a scattering state is used to describe the unbound continuum state. For transfer reactions both of the $\phi$ correspond to bound states. Their external forms are [9]

$$
\phi_{l m}(\vec{r})=C_{l} \gamma i^{l+1} h_{l}^{+}(i \gamma r) Y_{l m}(\widehat{r}),
$$

where $\gamma=\sqrt{2 m|\epsilon|} / \hbar$ is determined by the separation energy $|\epsilon|$ and $\widehat{r}$ denotes the angular dependence of the vector $\vec{r}$. We use $h^{+}=i h^{(1)}$, where the $h^{(1)}$ are the spherical Bessel 
functions of the third kind [16], or Hankel functions, and $C_{l}$ is the asymptotic normalization coefficient (ANC) obtained by matching the calculated bound state solution to its external form.

As was shown in Ref. [17], introducing the double Fourier transforms of these bound state wave functions, defined by

$$
f_{l m}\left(x, k_{y}, k_{z}\right)=\int_{-\infty}^{\infty} \int_{-\infty}^{\infty} \exp \left[-i\left(k_{z} z+k_{y} y\right)\right] \phi_{l m}(\vec{r}) d z d y,
$$

allows Eq. (6) to be written as

$$
\begin{aligned}
A\left(b, l_{1} m_{1} l_{2} m_{2}\right)= & \frac{\hbar}{2 \pi m i v} \int_{-\infty}^{\infty} d k_{y} \sqrt{\xi^{2}+k_{y}^{2}} \\
& \times f_{l_{1} m_{1}}\left(b-x_{0}, k_{y}, k_{1}\right) f_{l_{2} m_{2}}^{*}\left(x_{0}, k_{y}, k_{2}\right),
\end{aligned}
$$

where

$$
\xi^{2}=k_{1}^{2}+\gamma_{i}^{2}=k_{2}^{2}+\gamma_{f}^{2},
$$

$x_{0}$ is the distance from the target to the $\Sigma$ surface, Fig. 1, and $k_{1}=\left[\epsilon_{f}-\epsilon_{i}-m v^{2} / 2\right] / \hbar v, \quad k_{2}=\left[\epsilon_{f}-\epsilon_{i}+m v^{2} / 2\right] / \hbar v$.

As was discussed in Refs. [9,10], $k_{1}\left(k_{2}\right)$ can be interpreted as the $z$-component of the momentum of the transferred particle in the projectile (target) rest frame, while $\xi$ as the magnitude of its transverse momentum.

When the wave functions assume their external forms then these $f_{l m}$ can be evaluated analytically and

$$
\begin{aligned}
f_{l_{1} m_{1}}\left(b-x_{0}, k_{y}, k_{z}\right) & =\frac{2 \pi}{\gamma_{i, x}} C_{l_{1}} \exp \left[-\gamma_{i, x}\left(b-x_{0}\right)\right] Y_{l_{1} m_{1}}\left(\widehat{\mathcal{K}}_{i}\right), \\
f_{l_{2} m_{2}}\left(x_{0}, k_{y}, k_{z}\right) & =\frac{2 \pi}{\gamma_{f, x}} C_{l_{2}} \exp \left[-\gamma_{f, x} x_{0}\right] Y_{l_{2} m_{2}}\left(\widehat{\mathcal{K}}_{f}\right) .
\end{aligned}
$$

Here we have introduced the (complex) vectors $\overrightarrow{\mathcal{K}}_{i}$ and $\overrightarrow{\mathcal{K}}_{f}$ with components

$$
\overrightarrow{\mathcal{K}}_{i}=\left(i \gamma_{i, x}, k_{y}, k_{z}\right), \quad \overrightarrow{\mathcal{K}}_{f}=\left(i \gamma_{f, x}, k_{y}, k_{z}\right),
$$

where

$$
\gamma_{i, x}^{2}=k_{z}^{2}+k_{y}^{2}+\gamma_{i}^{2}, \quad \gamma_{f, x}^{2}=k_{z}^{2}+k_{y}^{2}+\gamma_{f}^{2},
$$

and the spherical harmonics of complex argument are understood in terms of the (homogeneous) solid harmonic polynomials

$$
\left(i \gamma_{f}\right)^{l} Y_{l m}\left(\widehat{\mathcal{K}}_{f}\right)=\mathcal{Y}_{l m}\left(i \gamma_{f, x}, k_{y}, k_{z}\right) .
$$

Upon introduction of the explicit forms of Eq. (12), the transfer amplitude can be stated analytically [10], as

$$
\begin{aligned}
A\left(b, l_{1} m_{1} l_{2} m_{2}\right)= & \frac{4 \pi \hbar}{m i v} C_{l_{1}} C_{l_{2}}^{*} K_{m_{1}-m_{2}}(\xi b) \\
& \times Y_{l_{2} m_{2}}^{*}\left(\beta_{2}, 0\right) Y_{l_{1} m_{1}}\left(\beta_{1}, \pi\right),
\end{aligned}
$$

where $K_{m_{1}-m_{2}}$ is the modified Bessel function of order $m_{1}-m_{2}$ [16] and $\beta_{1}$ and $\beta_{2}$ are the (complex) polar angles

$$
\cos \beta_{1}=-i k_{1} / \gamma_{i}, \quad \cos \beta_{2}=-i k_{2} / \gamma_{f} .
$$

Here $Y_{l_{2} m_{2}}^{*}\left(\beta_{2}, 0\right)$ should be understood as $\left[Y_{l_{2} m_{2}}\left(\beta_{2}, 0\right)\right]^{*}$.

\section{B. Breakup cross section}

In the breakup case the final state external wave function is no longer defined by Eq. (7) but by a scattering state with an ingoing spherical-wave boundary condition, $\Psi_{\vec{k}_{f}}^{(-)}[18]$ with $\vec{k}_{f}$ the asymptotic neutron-target relative motion wave vector. The matrix element that provides the transition amplitude is now

$$
A\left(b, l_{1} m_{1} \vec{k}_{f}\right)=\frac{1}{i \hbar} \int_{-\infty}^{\infty} d t\left\langle\Psi_{\vec{k}_{f}}^{(-)}\left|V_{\mathrm{cn}}\right| \Psi_{i}\right\rangle .
$$

Using the time-reversal relationship $\left[\Psi_{\vec{k}_{f}}^{(-)}(\vec{r})\right]^{*}=\Psi_{-\vec{k}_{f}}^{(+)}(\vec{r})$,

$$
\begin{aligned}
{\left[\Psi_{\vec{k}_{f}}^{(-)}(\vec{r})\right]^{*}=} & 4 \pi \sum_{l_{2} m_{2}}(-i)^{l_{2}} Y_{l_{2} m_{2}}^{*}\left(\widehat{k}_{f}\right) Y_{l_{2} m_{2}}(\widehat{r}) \frac{i}{2} \\
& \times\left[h_{l_{2}}^{-}\left(k_{f} r\right)-S_{l_{2}} h_{l_{2}}^{+}\left(k_{f} r\right)\right],
\end{aligned}
$$

where $S_{l_{2}}$ is the neutron-target $S$ matrix, $S_{l_{2}}=\exp \left(2 i \delta_{l_{2}}\right)$. The same procedure as in the transfer case can be carried out for breakup, i.e., to express amplitude Eq. (18) as the flux through the intermediate $\Sigma$ surface on which the wave functions take their external forms [13]. The breakup amplitude becomes

$$
\begin{aligned}
A\left(b, l_{1} m_{1} \vec{k}_{f}\right)= & \frac{16 \pi^{2} C_{l_{1}} \hbar}{m v k_{f}} \sum_{l_{2} m_{2}} \mathcal{J}_{l_{2}} K_{m_{1}-m_{2}}(\eta b) Y_{l_{2} m_{2}}\left(\widehat{k}_{f}\right) \\
& \times Y_{l_{2} m_{2}}^{*}\left(\beta_{2}, 0\right) Y_{l_{1} m_{1}}\left(\beta_{1}, \pi\right),
\end{aligned}
$$

where we have introduced $\mathcal{J}_{l_{2}}=\left[1-S_{l_{2}}\right] / 2$, and where $\eta$, the breakup analogue of $\xi$ of Eq. (10), is

$$
\eta^{2}=k_{1}^{2}+\gamma_{i}^{2}=k_{2}^{2}-k_{f}^{2},
$$

and now

$$
\cos \beta_{2}=k_{2} / k_{f} .
$$

Introducing the breakup amplitude of Eq. (20) in Eqs. (1) and (2), the TC breakup cross section is

$$
\begin{aligned}
\frac{d \sigma}{d k_{1}}= & \frac{32 \pi}{\left(2 l_{1}+1\right)} \frac{\mu_{i}}{\mu_{f}} \frac{\hbar\left|C_{l_{1}}\right|^{2}}{m v k_{f}} \int d \vec{b} P_{\mathrm{el}}(b) \\
& \times \sum_{l_{2} m_{1} m_{2}}\left|K_{m_{1}-m_{2}}(\eta b) \mathcal{J}_{l_{2}} Y_{l_{2} m_{2}}^{*}\left(\beta_{2}, 0\right) Y_{l_{1} m_{1}}\left(\beta_{1}, \pi\right)\right|^{2} .
\end{aligned}
$$

In the absence of spin-dependent distortions, the spin of the transferred neutron is simply a spectator in the reaction. Thus, even if the initial bound state total angular momentum $j_{1}$ does not appear explicitly in Eq. (23), there is an implied $j_{1}$ dependence through the bound state ANC $C_{l_{1}}$. Herein, we refer to Eq. (23) as the full, or exact, Bessel function form of the cross section.

In Ref. [8] it was also shown that an approximate treatment of the cross section for inelastic breakup, also referred to as the stripping mechanism, and taking account of all inelastic excitations of the target, can be included by redefining

$$
\left|\mathcal{J}_{l_{2}}\right|^{2}=\left[\left\{1-\left|S_{l_{2}}\right|^{2}\right\}+\left|1-S_{l_{2}}\right|^{2}\right] / 4 \text {. }
$$

The elastic and inelastic breakup terms are represented by $\left|1-S_{l_{2}}\right|^{2}$ and $1-\left|S_{l_{2}}\right|^{2}$, respectively. 


\section{APPROXIMATIONS TO THE TC AMPLITUDES}

With the purpose of carrying these analytical expressions as far as possible, and being assisted by the large typical $\xi b$ (or $\eta b$ ) values implied by transfer between heavy nuclear cores, further simplifications of Eq. (23) have been carried out in the literature. These approximations are summarized in this section.

\section{A. Truncated Bessel function expansion approximation}

As discussed above, the TC model was originally developed from consideration of reactions involving particle transfer between heavy ion cores [19,20]. A characteristic of such heavy (large) nuclear systems is that $P_{\mathrm{el}}(b)$ is significantly different from zero only for such large values of $b$ that $\eta b$ is large. In these cases the $K_{m_{1}-m_{2}}(\eta b)$ Bessel function can be approximated by its asymptotic expansion [16]

$$
\begin{aligned}
K_{m_{1}-m_{2}}(\eta b) \simeq & \sqrt{\frac{\pi}{2 \eta b}} \exp (-\eta b) \\
& \times\left(1+\frac{\mu-1}{8 \eta b}+\frac{(\mu-1)(\mu-9)}{2(8 \eta b)^{2}}+\cdots\right),
\end{aligned}
$$

where $\mu=4\left(m_{1}-m_{2}\right)^{2}$. If only the leading (zeroth-order) term is retained [21] then its dependence on $m_{1}-m_{2}$ disappears, and the sums over $m_{1}$ and $m_{2}$ in Eq. (23) can be carried out using the identity

$$
\sum_{m}\left|Y_{l m}(\theta, \phi)\right|^{2}=\frac{(2 l+1)}{4 \pi} P_{l}(\cos \theta)
$$

where $P_{l}$ is the Legendre Polynomial of order $l$. Eq. (23) then reads

$$
\begin{aligned}
\frac{d \sigma}{d k_{1}} \approx & \frac{\mu_{i}}{\mu_{f}} \frac{\hbar\left|C_{l_{1}}\right|^{2}}{m v k_{f}} \int d \vec{b} P_{\mathrm{el}}(b) \frac{\exp (-2 \eta b)}{\eta b} P_{l_{1}}\left(\cos \beta_{1}\right) \\
& \times \sum_{l_{2}}\left(2 l_{2}+1\right)\left|\mathcal{J}_{l_{2}}\right|^{2} P_{l_{2}}\left(\cos \beta_{2}\right)
\end{aligned}
$$

as was obtained in Refs. [9,22]. We refer to this as the zeroth-order TC approximation.

\section{B. $M$-function approximation}

In an attempt to extend the applications of the formalism to lighter nuclei, but retaining the analytical clarity, in Ref. [11] the following $M$-function approximation was developed for transfer and later applied to breakup studies. Eq. (23) can be written as

$$
\begin{aligned}
\frac{d \sigma}{d k_{1}}= & \frac{8 \pi}{\left(2 l_{1}+1\right)} \frac{\mu_{i}}{\mu_{f}} \frac{\hbar\left|C_{l_{1}}\right|^{2}}{m v k_{f}} \int d \vec{b} P_{\mathrm{el}}(b) \\
& \times \sum_{l_{2} m_{1} m_{2}}\left|\mathcal{J}_{l_{2}} \int_{-\infty}^{\infty} d k_{y} \frac{\exp \left(-\gamma_{x} b\right)}{\gamma_{x}} Y_{l_{1} m_{1}}\left(\widehat{\mathcal{K}}_{i}\right) Y_{l_{2} m_{2}}^{*}\left(\widehat{\mathcal{K}}_{f}\right)\right|^{2},
\end{aligned}
$$

where $\overrightarrow{\mathcal{K}}_{f}=\left(i \gamma_{f, x}, k_{y}, k_{z}\right)$, Eq. (13), but now $\gamma_{f, x}^{2}=k_{z}^{2}+$ $k_{y}^{2}-k_{f}^{2}$. This can be rewritten

$$
\begin{aligned}
\frac{d \sigma}{d k_{1}}= & \frac{1}{2 \pi} \frac{\mu_{i}}{\mu_{f}} \frac{\hbar\left|C_{l_{1}}\right|^{2}}{m v k_{f}} \int d \vec{b} P_{\mathrm{el}}(b) \sum_{l_{2}}\left(2 l_{2}+1\right)\left|\mathcal{J}_{l_{2}}\right|^{2} \\
\times & {\left[\int d k_{y} \int d k_{y}^{\prime} \frac{\exp \left(-b\left[\gamma_{x}+\gamma_{x}^{\prime}\right]\right)}{\gamma_{x} \gamma_{x}^{\prime}}\right.} \\
& \times P_{l_{1}}\left(\frac{k_{y} k_{y}^{\prime}+k_{1}^{2}+\gamma_{x} \gamma_{x}^{\prime}}{\gamma_{i}^{2}}\right) \\
& \left.\times P_{l_{2}}\left(\frac{k_{y} k_{y}^{\prime}+k_{1}^{2}+\gamma_{x} \gamma_{x}^{\prime}}{k_{f}^{2}}\right)\right]
\end{aligned}
$$

where we have made use of the definitions of the complex angles, see Eqs. (17) and (22), and have defined $\gamma_{x}=$ $\sqrt{\eta^{2}+k_{y}^{2}}$ and $\gamma_{x}^{\prime}=\sqrt{\eta^{2}+k_{y}^{\prime 2}}$. Equation (29) can be approximated [11], in the limit that $\eta \gg k_{y}$, to give

$$
\begin{aligned}
\frac{d \sigma}{d k_{1}} \simeq & \frac{\mu_{i}}{\mu_{f}} \frac{\hbar\left|C_{l_{1}}\right|^{2}}{m v k_{f}} \int d \vec{b} P_{\mathrm{el}}(b) \frac{\exp (-2 \eta b)}{\eta b} \\
& \times \sum_{l_{2}}\left(2 l_{2}+1\right)\left|\mathcal{J}_{l_{2}}\right|^{2} M\left(l_{1}, l_{2}\right),
\end{aligned}
$$

with

$$
\begin{aligned}
M\left(l_{1}, l_{2}\right)= & \frac{1}{\sqrt{\pi}} \int_{-\infty}^{\infty} d X \exp \left(-X^{2}\right) P_{l_{1}}\left(A_{i}+B_{i} X^{2}\right) \\
& \times P_{l_{2}}\left(A_{f}+B_{f} X^{2}\right)
\end{aligned}
$$

and where

$A_{i}=1+\frac{2 k_{1}^{2}}{\gamma_{i}^{2}}, B_{i}=\frac{2 \eta}{b \gamma_{i}^{2}}, \quad A_{f}=\frac{2 k_{2}^{2}}{k_{f}^{2}}-1, B_{f}=\frac{2 \eta}{b k_{f}^{2}}$.

\section{CHOICE OF MODEL PARAMETERS}

We now apply the theoretical formalisms developed in Secs. II and III to three specific knockout reaction transitions. All examples were the subject of recent experiments carried out at the National Superconducting Cyclotron Laboratory at Michigan State University [5,7]. These are the ${ }^{9} \mathrm{Be}\left({ }^{34} \mathrm{Si},{ }^{33} \mathrm{Si}+\gamma\right) X$ reaction at a beam energy of $73 \mathrm{MeV}$ per nucleon [5], for which the ground state to ground state one-neutron separation energy is $7.36 \mathrm{MeV}$, and the ${ }^{9} \mathrm{Be}\left({ }^{15} \mathrm{C},{ }^{14} \mathrm{C}+\gamma\right) X$ reaction at $54 \mathrm{MeV}$ per nucleon [7] with ground state separation energy of only $1.218 \mathrm{MeV}$. We consider also the ${ }^{15} \mathrm{C} \rightarrow{ }^{14} \mathrm{C}\left(1^{-}, 6.09 \mathrm{MeV}\right)$ transition with separation energy $7.37 \mathrm{MeV}$. The ground state transitions are $0^{+} \rightarrow 3 / 2^{+}$ and $1 / 2^{+} \rightarrow 0^{+}$, respectively. Our examples have been chosen to examine the sensitivity of approximations in the TC model calculations to both the nucleon orbital angular momentum and the separation energy.

As was discussed earlier, the TC model uses a description of the initial and final state wave functions of the transferred 
nucleon which are valid only in the external region. The separation energy is seen to enter the model through the reaction kinematics, the wave function matching conditions, Eq. (21), and the assumed external form of the single-particle overlap function (the spherical Hankel function) and its ANC, $C_{l}$. These $C_{l}$ are computed, using Eq. (7), by matching a Hankel function to the tails of the entrance channel bound state radial wave functions $\chi_{j l}(r)$ calculated in Woods-Saxon potentials with a fixed geometry $\left(r_{0}=1.25 \mathrm{fm}\right.$ and $\left.a=0.7 \mathrm{fm}\right)$, a spin-orbit potential of strength $6.0 \mathrm{MeV}$ and with the central potential depths adjusted to reproduce the required neutron separation energies. For the ${ }^{33} \mathrm{Si}$ (g.s.) transition, assuming a $0 d_{3 / 2}$ neutron configuration, then $C_{2}=2.92 \mathrm{fm}^{-1 / 2}$. For the $1 s_{1 / 2},{ }^{14} \mathrm{C}$ (g.s.) transition we obtain $C_{0}=1.47 \mathrm{fm}^{-1 / 2}$ while for the excited, $0 p_{3 / 2}{ }^{14} \mathrm{C}\left(1^{-}, 6.09 \mathrm{MeV}\right)$ neutron transition, $C_{1}=3.11 \mathrm{fm}^{-1 / 2}$. For the given parameters the depths of the potential wells are $46.48 \mathrm{MeV}$ for ${ }^{34} \mathrm{Si}$ and $49.32 \mathrm{MeV}$ and $45.27 \mathrm{MeV}$ for the g.s. and $1^{-}$transitions from ${ }^{15} \mathrm{C}$.

\section{A. Core-target interaction}

In the TC formalism, the interaction $V_{\mathrm{ct}}$ of the heavy projectile core, or residue, with the target nucleus does not affect the dynamics of the core motion during the reaction. The core is assumed to travel a straight line path at constant velocity. Rather, the effects of $V_{\mathrm{ct}}$ enter the formalism (semiclassically) through the associated probability of the passage of the core, in the elastic channel, past the target at an impact parameter $b$. This is the factor $P_{\mathrm{el}}(b)$ in Eq. (23). This is of course the square modulus of the core-target elastic $S$ matrix, $\left|\exp \left(2 i \delta_{L}\right)\right|^{2}$, entering through Eq. (2), but expressed as a function of the core impact parameter, $b=$ $(L+1 / 2) / K$. Since, theoretically, the peripheral nature of the reaction is dictated entirely by the absorptive content of this factor at small impact parameters, its spatial form is of some importance and must accurately reflect the empirical residue-target reaction cross section. Since the TC model assumes not only peripherality of the reaction, but that we are fully external in the wave functions of the transferred particle in the initial and final states, $P_{\mathrm{el}}(b)$ is instrumental in determining the actual active regions of radial overlap of these wave functions and hence the resulting cross sections.

To take proper account of the geometrical sizes of the core and target and their associated strong absorption radius, we construct $V_{\mathrm{ct}}$ by the double-folding of their matter densities with an effective nucleon-nucleon $(N N)$ interaction. The simple Gaussian effective $N N$ interaction used was chosen to reproduce measured heavy-ion cross section systematics in this energy and mass region within such double-folding model calculations $[23,24]$. The $P_{\mathrm{el}}(b)=\left|S_{\mathrm{ct}}(b)\right|^{2}$ were calculated from the complex double folding model $V_{\mathrm{ct}}$ using the eikonal approximation to the $S$ matrix, $S_{\mathrm{ct}}(b)$, e.g., [2].

The ${ }^{14} \mathrm{C}$ and ${ }^{33} \mathrm{Si}$ core matter densities were assumed to have Gaussian form factors with range parameters chosen to reproduce the empirically deduced root mean squared matter radii. These were $2.30 \mathrm{fm}[25]$ and $3.16 \mathrm{fm}$, respectively. The latter value was based on an assumed charge radius of $3.27 \mathrm{fm}$, estimated from comparisons with the stable $\mathrm{S}$ and $\mathrm{Si}$ isotopes

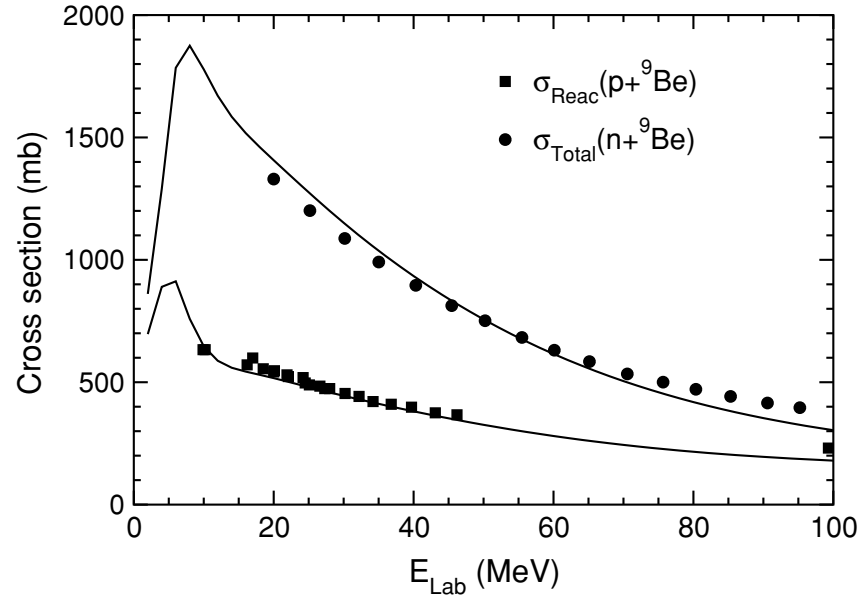

FIG. 2. The $p+{ }^{9} \mathrm{Be}$ reaction cross section and $n+{ }^{9} \mathrm{Be}$ total cross section predictions using the JLM folding model optical potential. The data are from Refs. [32] and [33], respectively.

[26]. The density used for the ${ }^{9} \mathrm{Be}$ target is detailed in the following subsection.

\section{B. Neutron-target interaction}

We described the neutron-target system using the nuclear matter effective $N N$ interaction of Jeukenne, Lejeune, and Mahaux (JLM) [27], based on the Reid hard-core free $N N$ interaction [28]. The resulting energy- and density-dependent effective interaction is used to calculate the nucleon optical potential on the finite target by single folding with its assumed one-body density, using the midpoint local density prescription [29]. This potential included the required effective mass correction to the imaginary part (Eq. (29) of Ref. [27]) discussed in Refs. [30,31]. We use the conventional scale factors for the computed real and imaginary parts of the optical potential, $\lambda_{V}=1.0$ and $\lambda_{W}=0.8$, required by an extended analysis of data on light and medium mass targets [29]. We take the ${ }^{9} \mathrm{Be}$ matter density $\rho(r)$ from Ref. [26], which is of the harmonic oscillator form

$$
\rho(r)=\rho_{0}\left[1+\alpha x^{2}\right] \exp \left(-x^{2}\right),
$$

with $x=r / a$. Given the values $a=1.77 \mathrm{fm}$ and $\alpha=$ 0.631 [26] then $\rho_{0}=0.1497 \mathrm{fm}^{-3}$. We assume that the neutron density is $\rho_{n}(r)=N \rho(r) / A$, and similarly for $\rho_{p}(r)$.

The predictions of the JLM model for the $p+{ }^{9} \mathrm{Be}$ reaction [32] and $n+{ }^{9} \mathrm{Be}$ [33] total cross sections are compared with available data in Fig. 2. The agreement is very reasonable in the energy range of the data, and that of importance to the beam energies of the reactions of interest. Since, at energies above $20 \mathrm{MeV}$, the Coulomb interaction is unimportant for our light ${ }^{9} \mathrm{Be}$ target, the $p+{ }^{9} \mathrm{Be}$ data provide support for the applicability of the JLM neutron-target optical potential and for the ${ }^{9} \mathrm{Be}$ density used. 


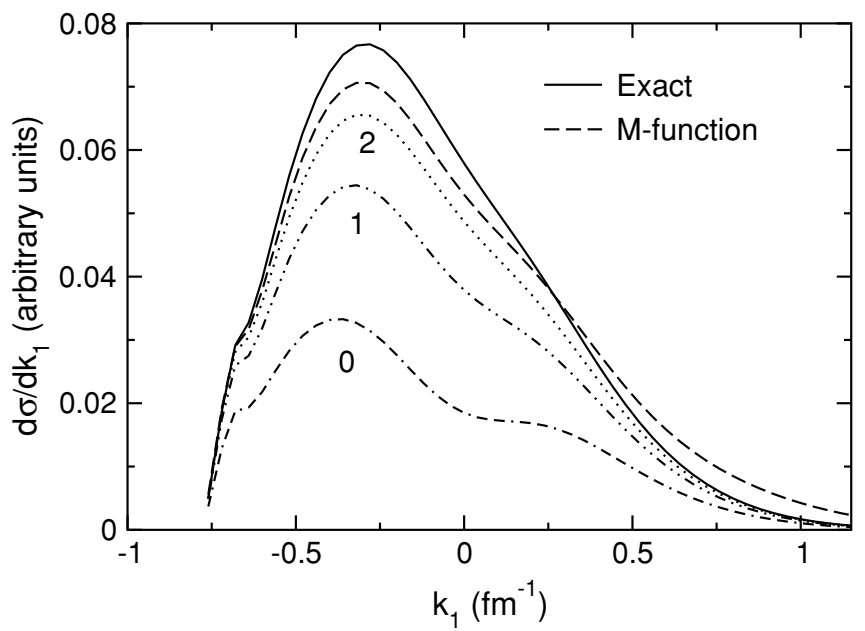

FIG. 3. $M$ function, zeroth order (labeled 0), and full Bessel function approximation calculations of $d \sigma / d k_{1}$ for the ${ }^{9} \mathrm{Be}\left[{ }^{34} \mathrm{Si}\right.$, ${ }^{33} \mathrm{Si}$ (g.s.) $] X$ reaction at $73 \mathrm{MeV}$ per nucleon. Also shown are the results including terms up to first (labeled 1) and second order (labeled 2) in the Bessel function expansion.

\section{RESULTS AND DISCUSSION}

Published elastic breakup and nucleon knockout cross sections from the TC model have usually been calculated [8,22] within the $M$-function approximation, Eq. (30), of Sec. III B. We were able to reproduce in detail the calculations presented in Ref. [5,22] with the parameters stated there. The more approximate approach, of retaining only the leading (zeroth-order) term of the asymptotic expansion of the Bessel function, Eq. (27), discussed in Sec. III A, was also used in some early papers, e.g. [8]. We are now in a position to compare the accuracy of these schemes using calculations that retain the full Bessel function, through Eq. (23). All the subsequent calculations are carried out using the common set of physical model parameters detailed in the previous section.

In Fig. 3 we present the zeroth-order, Eq. (27), $M$ function, Eq. (30), and full Bessel function, Eq. (23), approximations to $d \sigma / d k_{1}$ for the ${ }^{9} \mathrm{Be}\left[{ }^{34} \mathrm{Si},{ }^{33} \mathrm{Si}(\right.$ g.s. $\left.)\right] X$ reaction at $73 \mathrm{MeV}$ per nucleon. The corresponding integrated partial cross sections are presented in Table I. Figure 3 also shows the results of calculations including terms up to first and second order in the Bessel function expansion. So as not to needlessly complicate the figure, the third-order calculation is not shown. However, it essentially coincides with the $M$-function curve for small $k_{1}$ and with the full Bessel function result (the upper solid curve) in the large $k_{1}$ tail of this momentum distribution.

We comment also that the convergence of the $l_{2}$ (finalstate) partial wave sum in Eq. (23) is nontrivial and is very sensitive to the neutron-target interaction assumed. The rate of approach of $S_{l_{2}}$ to unity and $\left|\mathcal{J}_{l_{2}}\right|^{2}$ to zero with increasing $l_{2}$ in the summand is countered by rapidly increasing factors from the Bessel function and spherical harmonics. We found that the sum converges when using $V_{t n}$ models consistent with neutron-target data, such as the JLM optical model. Using optical model sets fitted to limited, low-energy data sets, such as from Ref. [34], the series was found to be divergent.

The $M$-function scheme is clearly a very significant improvement on the zeroth-order Bessel function approach. It is also clear that the different calculations are most sensitive to the different orders of approximation for $k_{1} \approx 0$. This is to be expected since the two approximate schemes are both large $\eta$ approximations and, for $k_{1}=0, \eta$ is a minimum, Eq. (10). As $k_{1}$ grows, all the Bessel function approximations are seen to come together.

Similarly, since $\eta$ also increases with increasing separation energy, Eq. (21), one might naively expect that the approximate TC models would agree better with the full Bessel function calculation for more tightly bound systems. However, the relevant expansion parameter is $\eta b$ and which values of $b$ contribute is determined by the product $P_{\text {el }}(b)\left|K_{m_{1}-m_{2}}(\eta b) Y_{l_{1} m_{1}}\left(\beta_{1}, \pi\right)\right|^{2}$. As is evident from Fig. 1, in the large $\left|\epsilon_{i}\right|$, tight binding limit only those $b$ values from near-grazing core-target collisions will contribute. The underlying TC requirement of an intermediate, external $\Sigma$ surface then suggests that all calculations are less valid in this regime. In contrast, smaller $\left|\epsilon_{i}\right|$ and greater spatial extension of the exterior wave function favors contributions from larger $b$. This sensitivity of the different (external) TC model approximations to the neutron separation energy is illustrated by carrying out a second (model) calculation in which the ${ }^{33} \mathrm{Si}$-neutron separation energy is arbitrarily reduced to just $0.5 \mathrm{MeV}$, from 7.36 MeV. We note that in this case, Fig. 4, the $M$-function approximation is actually further from the converged Bessel function calculation than the second-order

TABLE I. Calculated and experimental one-neutron knockout partial cross sections (in mb) on a ${ }^{9} \mathrm{Be}$ target. Calculations are the single-particle cross sections (for unit spectroscopic factors) when using the full semiclassical Bessel function amplitude of Eq. (23), the $M$-function approximation of Eq. (30) and the zeroth-order term Bessel function approximation of Eq. (27). The parameters used for each transition are detailed in the text and are the same for each of the TC calculation schemes. The deduced TC spectroscopic factors $S_{T C}$ from the full Bessel function calculations are also shown.

\begin{tabular}{lccccc}
\hline \hline Transition & $\begin{array}{c}\text { Full Bessel } \\
(\mathrm{mb})\end{array}$ & $\begin{array}{c}M \text { function } \\
(\mathrm{mb})\end{array}$ & $\begin{array}{c}\text { Zeroth order } \\
(\mathrm{mb})\end{array}$ & $\begin{array}{c}\text { Experiment } \\
(\mathrm{mb})\end{array}$ & $S_{\mathrm{TC}}$ \\
\hline${ }^{34} \mathrm{Si} \rightarrow{ }^{33} \mathrm{Si}($ g.s. $)$ & 28 & 26 & 10 & $67(10)$ & $2.4(4)$ \\
${ }^{15} \mathrm{C} \rightarrow{ }^{14} \mathrm{C}($ g.s. $)$ & 191 & 213 & 192 & $109(13)$ & $0.57(1)$ \\
${ }^{15} \mathrm{C} \rightarrow{ }^{14} \mathrm{C}\left(1^{-}, 6.09 \mathrm{MeV}\right)$ & 68 & 66 & 35 & $22(3)$ & $0.32(3)$ \\
\hline \hline
\end{tabular}




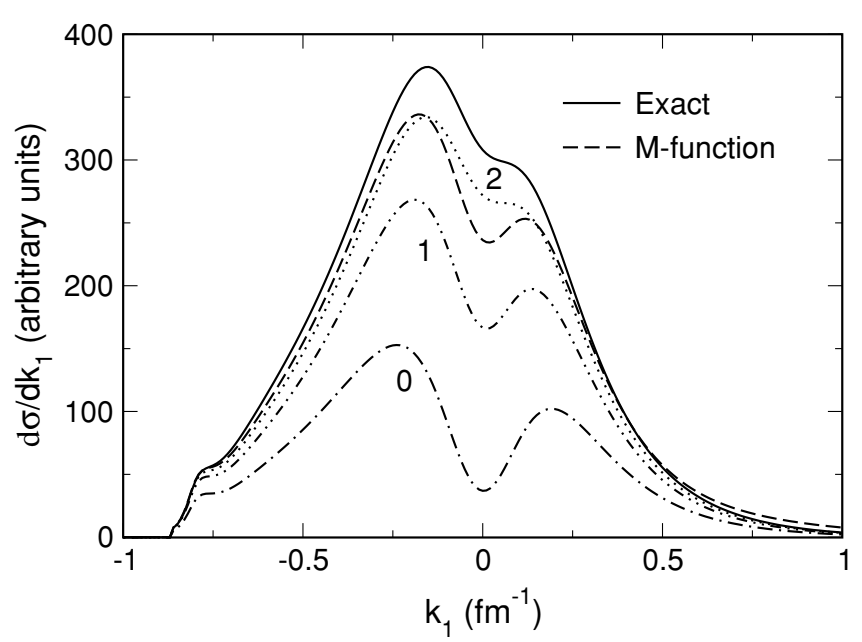

FIG. 4. As for Fig. 3 except that the ${ }^{33} \mathrm{Si}+$ neutron separation energy has been reduced to $0.5 \mathrm{MeV}$.

calculation, whereas in the more tightly bound system the $M$ function was consistent with the third-order approximation, Fig. 3. The calculations for both $\epsilon_{i}$ show that the zeroth-order approximation misses most of the cross section strength. This dependence of the accuracy of the $M$-function approximation on the separation energy may have spectroscopic implications when comparisons are made with experimental data.

As can also be seen from Eq. (11), and is evident in our Fig. 3, all of the TC models have a low $k_{1}$ cutoff. This negative $k_{1}$ bound is imposed by the $\epsilon_{f} \rightarrow 0$ limit in the numerator of the expression

$$
k_{1}=\left[\epsilon_{f}+\left|\epsilon_{i}\right|-m v^{2} / 2\right] / \hbar v
$$

and is seen to intrude within the physical region of the predicted and measured momentum distributions in cases where the $d \sigma / d k_{1}$ distribution is broad, i.e., those cases with tight binding and/or non- $s$-state transitions. This cutoff is an artifact of the model and results from its use of both semiclassical dynamics and of external wave functions, which have increased high momentum components compared with realistic wave functions.

Another noticeable feature of the results in Figs. 3 and 4, which will be also evident for the ${ }^{14} \mathrm{C}\left(1^{-}\right)$transition, Fig. 6 , is that the $M$-function scheme yields an enhanced tail to the cross section at the larger $k_{1}$. To understand this feature the nature of the $M$-function scheme must be considered. As was discussed in Ref. [11], the $M$ function arises from expanding (in $\eta$ ) only some of the terms that arise from the square of the integral form of the Bessel function. Whether this scheme will then yield bigger or smaller results than from the different orders of the asymptotic expansion will depend on $\eta$. Figures 3 and 4 show that with increasing $k_{1}$ the $M$ function $d \sigma / d k_{1}$ is largest. The $M$-function approximation thus exaggerates the high- $k_{1}$ tail of $d \sigma / d k_{1}$, that is, the low-momentum region of the predicted core momentum distribution $d \sigma / d p_{\|}$, Fig. 5. The core parallel momentum $\left(p_{c}\right)$ dependence in the projectile rest frame is obtained from $d \sigma / d k_{1}$, assuming $p_{c}=-k_{1}$, and is then boosted into the laboratory frame to give $d \sigma / d p_{\|}$.

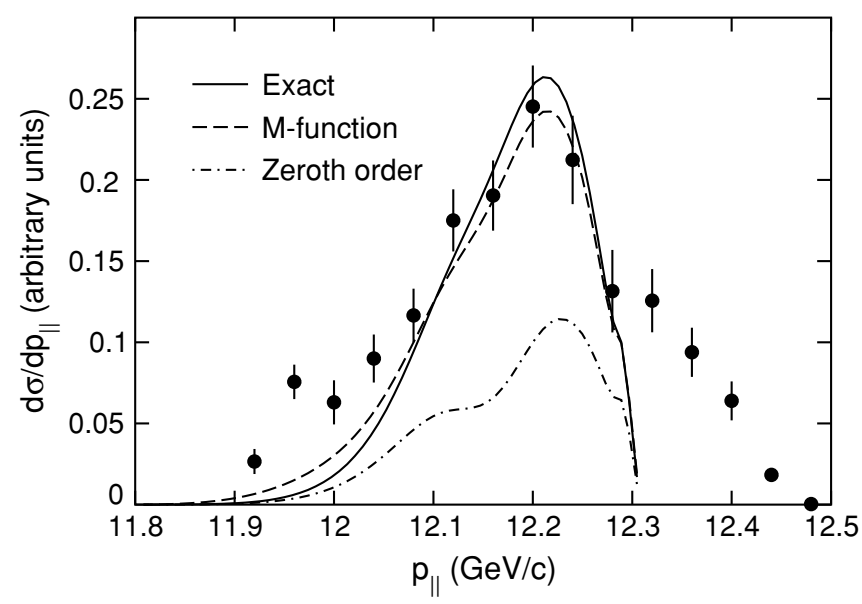

FIG. 5. $M$ function, zeroth order, and full Bessel function approximation calculations of the ${ }^{33} \mathrm{Si}$ (g.s.) cross section momentum distribution in the ${ }^{9} \mathrm{Be}\left({ }^{34} \mathrm{Si},{ }^{33} \mathrm{Si}\right) X$ reaction at $73 \mathrm{MeV}$ per nucleon. The experimental data are from Ref. [5].

Obviously the particular TC approximation used has an effect on the calculated single particle partial cross sections and will affect any spectroscopic strengths deduced from comparisons with data. Table I shows the values of the integrated partial cross sections from each of the three approaches for the two nuclei and three transitions considered. Except for the weakly-bound ${ }^{14} \mathrm{C}(\mathrm{g} . \mathrm{s})$ transition, the zeroth-order scheme predicts single-particle cross sections that are of order one-half those of the improved, higher-order approaches. Although the $M$-function scheme predicts somewhat different core momentum distributions, incorrectly moving cross section strength to lower values of $p_{\|}$, it nevertheless calculates very similar integrated partial cross sections to the full Bessel function approach.

Figures 5, 6, and 7 now compare the calculated and experimental core momentum distributions for the ${ }^{33} \mathrm{Si}$ (g.s.)

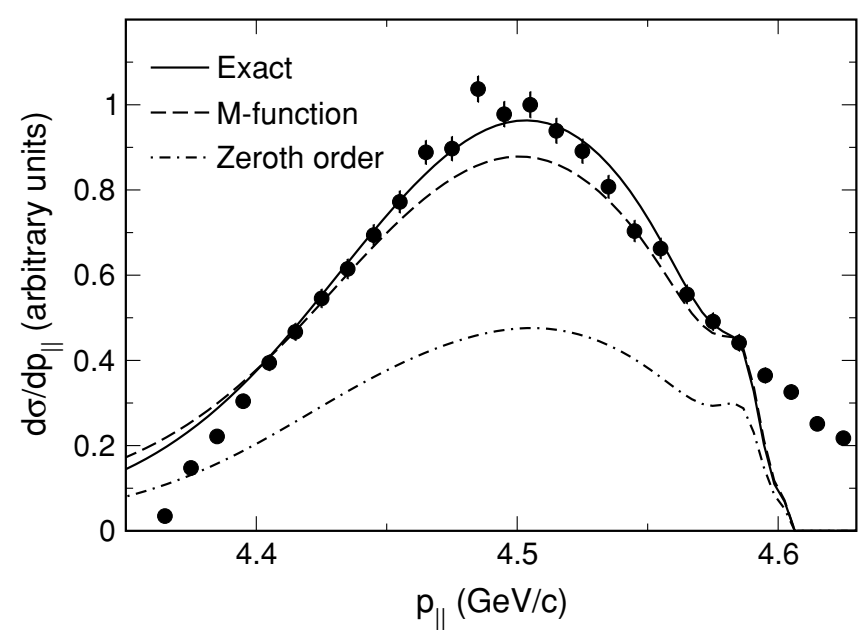

FIG. 6. $M$ function, zeroth order, and full Bessel function approximation calculations of the ${ }^{14} \mathrm{C}\left(1^{-}\right)$cross section momentum distribution in the ${ }^{9} \mathrm{Be}\left({ }^{15} \mathrm{C},{ }^{14} \mathrm{C}\right) X$ reaction at $54 \mathrm{MeV}$ per nucleon. The experimental data are from Ref. [7]. 


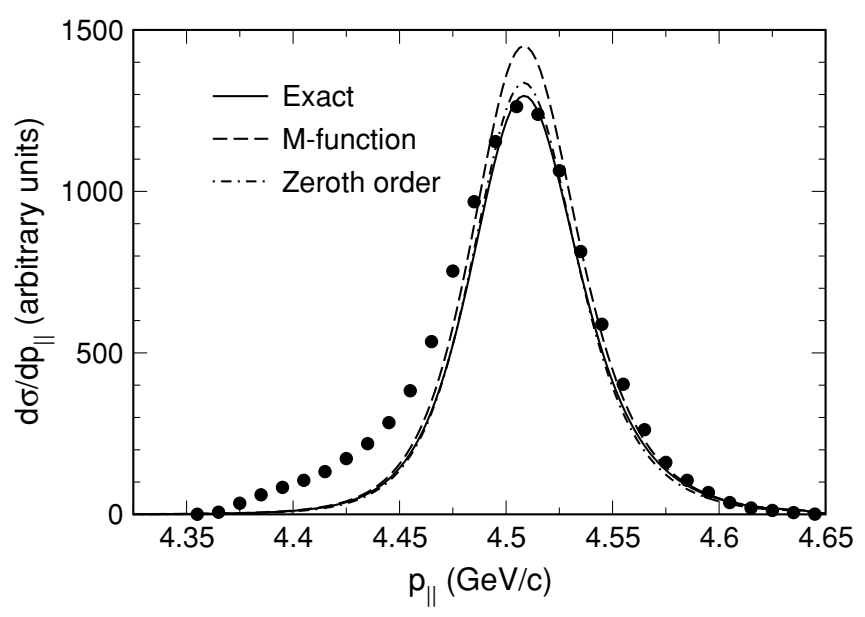

FIG. 7. $M$ function, zeroth order, and full Bessel function approximation calculations of the ${ }^{14} \mathrm{C}$ (g.s.) cross section momentum distribution in the ${ }^{9} \mathrm{Be}\left({ }^{15} \mathrm{C},{ }^{14} \mathrm{C}\right) X$ reaction at $54 \mathrm{MeV}$ per nucleon. The experimental data are from Ref. [7]

[5], ${ }^{14} \mathrm{C}\left(1^{-}\right)$, and ${ }^{14} \mathrm{C}$ (g.s) [7] transitions. In each case the full Bessel function calculations have been scaled to reproduce the peak of the momentum distribution. The more approximate calculations have been scaled by the same factor so that they can be compared in the figures. The experimental (integrated) partial cross sections have been divided by those of the full Bessel function model, shown in Table I, to deduce the TC scaling (or spectroscopic) factors $S_{T C}$, also collected there.

The shapes of the momentum distributions are, in general, poorly described. In the ${ }^{14} \mathrm{C}$ cases, the asymmetry in the g.s. transition data is not reproduced, while for the $1^{-}$transition the asymmetry in the TC calculation is not evident in the data. Figures 6 and 7 can also be compared directly with the analogous curves in Fig. 2 of Ref. [7]. Those calculations used the all-order, non-DWBA, eikonal theory, supplemented for the ground state transition by a fully-dynamical coupled channels calculation of the diffractive component, and used the same physical model inputs as were discussed in Sec. IV. There, the calculated ${ }^{14} \mathrm{C}$ single-particle partial cross sections were $\sigma_{s p}$ (g.s.) $=119 \mathrm{mb}$ and $\sigma_{s p}\left(1^{-}\right)=28 \mathrm{mb}$, with deduced spectroscopic factors close to unity and close to the shell model values.

Similarly, for the ${ }^{34} \mathrm{Si}$ transition, Fig. 5 , the TC yields $\sigma_{\mathrm{sp}}=$ $28 \mathrm{mb}$, almost a factor of 2 bigger than the $(15 \mathrm{mb})$ value obtained using eikonal theory [5]. The momentum distribution reproduces the data only in the immediate vicinity of the peak, and some asymmetry can be seen. However, the agreement between experiment and calculation quickly disappears for momenta away from those of the beam velocity, where the momentum distribution predicted by Eq. (23) falls far more quickly than the experimental data.

\section{CONCLUDING REMARKS}

The transfer-to-the-continuum direct reaction model provides an approximation to the DWBA description of elastic breakup of a projectile, based on a [nucleon+core]+target three-body model. The TC model has been shown to allow approximate calculations of both the elastic breakup and one-nucleon stripping cross sections, of relevance to recent knockout reaction studies. Previously, additional simplifying approximations have been used, allowing essentially analytical forms for these cross sections. In the present work we have calculated the TC amplitude more accurately, necessitating a numerical approach, but avoiding the need for the usuallyassumed simplifications.

In an attempt to clarify the accuracy of these TC model variants we have compared the theoretical predictions with and without the often-made large $\eta$ simplifications. In particular we have presented theoretical comparisons for three reactions with different $l$ and neutron separation energies. For all calculations the two-body interactions have been chosen to be consistent with the nucleon-target and core-target cross section systematics, as were used in recent eikonal model analyses.

Our results show that the predictions of the approximate versions of the TC theory are similar only for neutron removal from the $l=0,{ }^{15} \mathrm{C}$ neutron halo system, with small neutron separation energy. The $M$-function approximation is found to reliably reproduce the integrated partial cross sections of the more accurate exact Bessel function calculations, but to distort the momentum distribution, by moving strength to lower $p_{\|}$. For all the transitions studied, the integrated partial cross sections are significantly larger than those calculated using the all-order eikonal theory. In the present calculations spin-dependent distortions were excluded. Their effects on TC calculations of both cross sections and spin observables will be the subject of a subsequent paper.

\section{ACKNOWLEDGMENTS}

This work was supported by the United Kingdom Engineering and Physical Sciences Research Council (EPSRC) through Grant No. GR/M82141.
[1] P. G. Hansen and B. M. Sherrill, Nucl. Phys. A693, 133 (2001).

[2] P. G. Hansen and J. A. Tostevin, Ann. Rev. Nucl. Part. Sci. 53, 219 (2003).

[3] D. Cortina-Gil et al., Phys. Lett. B529, 36 (2002).

[4] E. Sauvan et al., Phys. Lett. B491, 1 (2000).

[5] J. Enders et al., Phys. Rev. C 65, 034318 (2002).

[6] V. Maddalena et al., Phys. Rev. C 63, 024613 (2001).
[7] J. A. Tostevin, D. Bazin, B. A. Brown, T. Glasmacher, P. G. Hansen, V. Maddalena, A. Navin, and B. M. Sherrill Phys. Rev. C 66, 024607 (2002).

[8] A. Bonaccorso and D. M. Brink, Phys. Rev. C 38, 1776 (1988).

[9] A. Bonaccorso, D. M. Brink, and L. Lo Monaco, J. Phys. G 13, 1407 (1987).

[10] L. Lo Monaco and D. M. Brink, J. Phys. G 11, 935 (1985). 
[11] F. Stancu and D. M. Brink, Phys. Rev. C 32, 1937 (1985).

[12] H. Hashim and D. M. Brink, J. Phys. G 11, 107 (1988).

[13] H. Hasan and D. M. Brink, J. Phys. G 4, 1573 (1978).

[14] D. M. Brink, in Nuclear Physics with Mesons and Heavy Ions, Les Houches 1977, edited by R. Balian et al. (North-Holland, Amsterdam, 1978), p. 48.

[15] E. Merzbacher, Quantum Mechanics, 3rd ed. (Wiley, New York, 1998), p. 145.

[16] M. Abramowitz and I. Stegun, Handbook of Mathematical Functions (Dover, New York, 1970), p. 437.

[17] A. Bonaccorso, G. Piccolo, and D. M. Brink, Nucl. Phys. A441, 555 (1985).

[18] R. C. Johnson, in An advanced course in modern nuclear physics, Lecture notes in Physics 581, edited by J. M. Arias and M. Lozano (Springer-Verlag, Berlin, 2001), p. 259.

[19] A. Bonaccorso and D. M. Brink, Phys. Rev. C 44, 1559 (1991).

[20] A. Bonaccorso and D. M. Brink, Phys. Rev. C 46, 700 (1992).

[21] A. Bonaccorso and D. M. Brink, Phys. Rev. C 58, 2864 (1998).
[22] A. Bonaccorso, Phys. Rev. C 60, 054604 (1999).

[23] J. A. Tostevin, J. Phys. G 25, 735 (1999).

[24] S. Kox et al., Phys. Rev. C 35, 1678 (1987).

[25] A. Ozawa et al., Nucl. Phys. A693, 32 (2001).

[26] C. W. De Jager et al., At. Data Nucl. Data Tables 14, 479 (1974).

[27] J. P. Jeukenne, A. Lejeune, and C. Mahaux, Phys. Rev. C 16, 80 (1977).

[28] R. V. Reid, Jr., Ann. Phys. (NY) 50, 411 (1968).

[29] J. S. Petler, M. S. Islam, R. W. Finlay, and F. S. Dietrich, Phys. Rev. C 32, 673 (1985).

[30] J. W. Negele and K. Yazaki, Phys. Rev. Lett. 47, 71 (1981).

[31] S. Fantoni, B. L. Friman, and V. Pandharipande, Phys. Lett. B104, 89 (1981).

[32] W. Bauhoff et al., At. Data Nucl. Data Tables 35, 429 (1986).

[33] R. W. Finlay, W. P. Abfalterer, G. Fink, E. Montei, T. Adami, P. W. Lisowski, G. L. Morgan, and R. C. Haight, Phys. Rev. C 47, 237 (1993).

[34] J. H. Dave and C. R. Gould, Phys. Rev. C 28, 2212 (1983). 